\title{
In situ TEM imaging of Nanoparticles interacting with Glioblastoma Stem Cells
}

\author{
Elliot S. Pohlmann ${ }^{1}$, Kaya Patel ${ }^{1}$, Sujuan Guo ${ }^{1}$, Madeline J. Dukes ${ }^{2}$, Zhi Sheng ${ }^{1}$, and Deborah F. Kelly ${ }^{1}$ \\ 1. Virginia Tech Carilion School of Medicine and Research Institute, Roanoke, VA 24016, USA. \\ 2. Applications Science, Protochips, Inc., Raleigh, NC, 97606, USA.
}

Modern cancer research is embracing alternative drug delivery vehicles to treat human tumors that are difficult to manage by conventional therapies. These systems often include, but are not limited to liposomal formulations, polymer coatings, gold nanoparticles (NPs), or combinatorial reagents. Gold NPs present an attractive platform for both therapeutic and medical imaging purposes as they have high contrast and small dimensions (typically $<100 \mathrm{~nm}$ ) [1,2]. As NP-based therapies are currently being used in clinical trials to treat solid tumors [3], this creates a major impetus to closely monitor their use and effectiveness. Current measurements are limited to the level of tissue accumulation while information concerning NPs interacting with individual cells remains missing from these analyses. As such, the development of new technologies to visualize and directly assess the behavior of NP-based therapies at the molecular level may contribute essential information toward evaluating therapeutic delivery, uptake, and efficacy.

To address this issue, we have recently engineered a new system to perform real-time visual assessments of NPs interacting with individual cells while contained in liquid. Here, we present the first real-time information of gold nanorods interacting with glioblastoma stem cells (GSCs). We elected to use these cells because (1) glioblastomas are a lethal threat to all patients diagnosed with these tumors; (2) malignant brain tumors are difficult to treat by conventional therapies, due to the GSCs that populate the tumors [4]; and (3) NP-based assessment for therapeutic purposes are currently ill-defined.

First, we enriched for GSCs from a mixed population of glioblastoma cells (GS9.6 line) based on the presence of the NOTCH1 receptor. GS9-6/NOTCH1+ cells were then tethered to silicon nitride microchips containing integrated microwells and coated with antibodies against the NOTCH1 receptor (Figure 1). The tethered cells were treated with PVP-coated gold nanorods (Nanopartz, Inc.) or aqueous solution lacking nanorods as a negative control. The samples were sealed in a Poseidon specimen holder (Protochips, Inc.) and imaged using in situ Transmission Electron Microscopy (TEM) protocols. Realtime recordings of the gold nanorods interacting at the cell membrane and within the cells (Figure 2) were acquired under low-dose conditions at $120 \mathrm{kV}$ using TEM. Contour plots of individual images acquired at various time intervals illustrated the dynamic movements of the nanorods with respect to the cell membranes. Therefore, our work shows the first real-time movements of gold NPs interacting with GSCs in a liquid environment using In Situ TEM. These advancements provide a new basis for directly evaluating the effects of NP therapeutics on individual cancer cells at the molecular level.

\section{References:}

[1] Chow, E. K., and D. Ho, Science Trans. Med. 5 (2013), pp. 216 rv214.

[2] Rink, J. S., Plebanek, M. P., Tripathy, S., and C. S. Thaxton, Current Opin. in Onco. 25 (2012), pp. 646-651.

[3] Yasun, E., Kang, H., Erdal, H., Cansiz, S., Ocsoy, I., Huang, Y. F., and W. Tan, Interface Focus 3 (2013), pp. 20130006.

[4] Stopschinski, B. E., Beier, C. P., and D. Beier, Cancer Letters 338 (2013), pp. 32-40. 

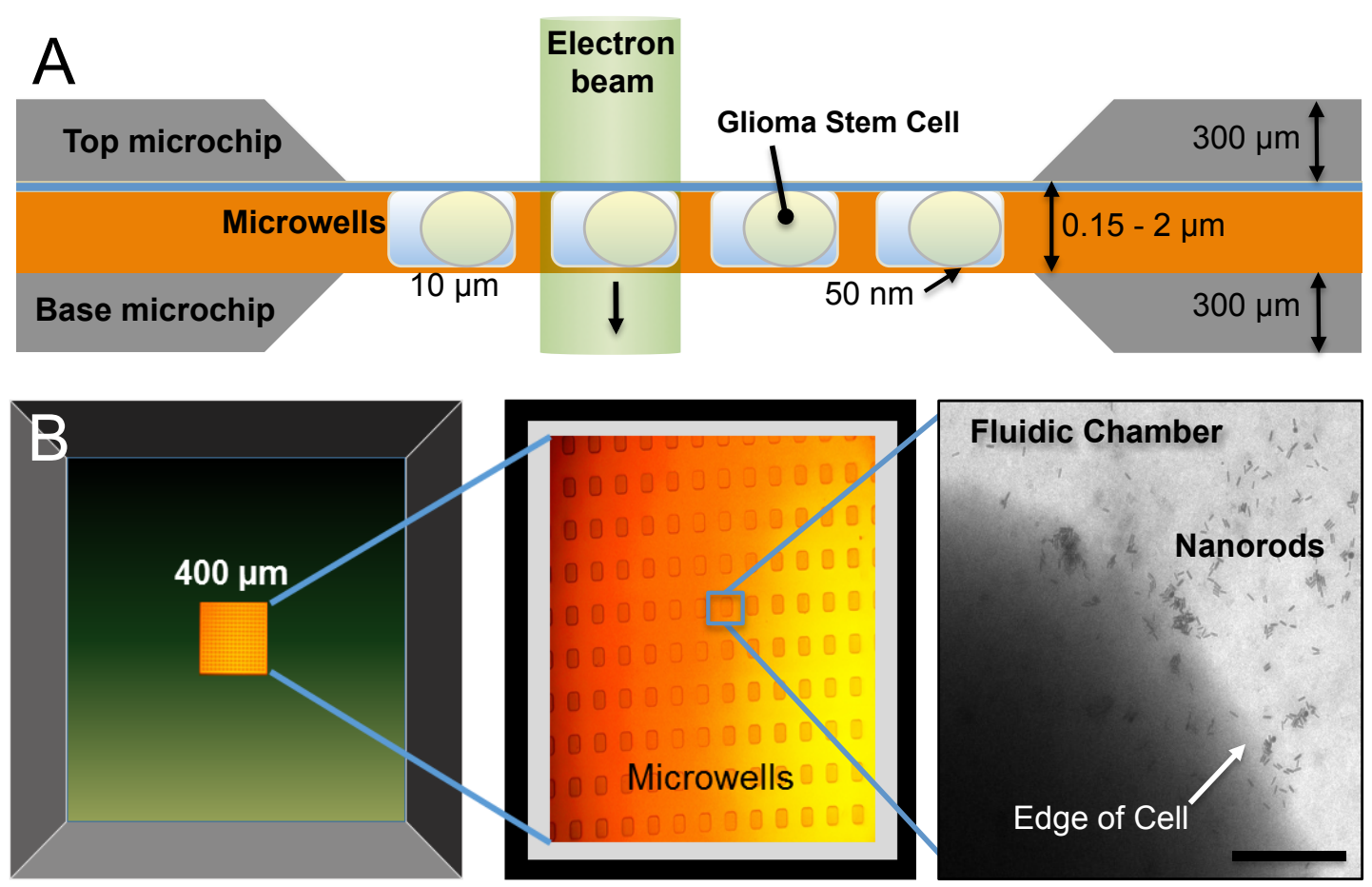

Figure 1. (A) Schematic to demonstrate a cross-sectional view of the microfluidic system containing GS9-6/NOTCH1+ glioma cells while positioned in the TEM. (B) Microchips with integrated microwells were used to specifically tether GS9-6/NOTCH1+ GSCs treated with gold nanorods within the fluidic chamber of the Poseidon specimen holder. Scale bar is $500 \mathrm{~nm}$.
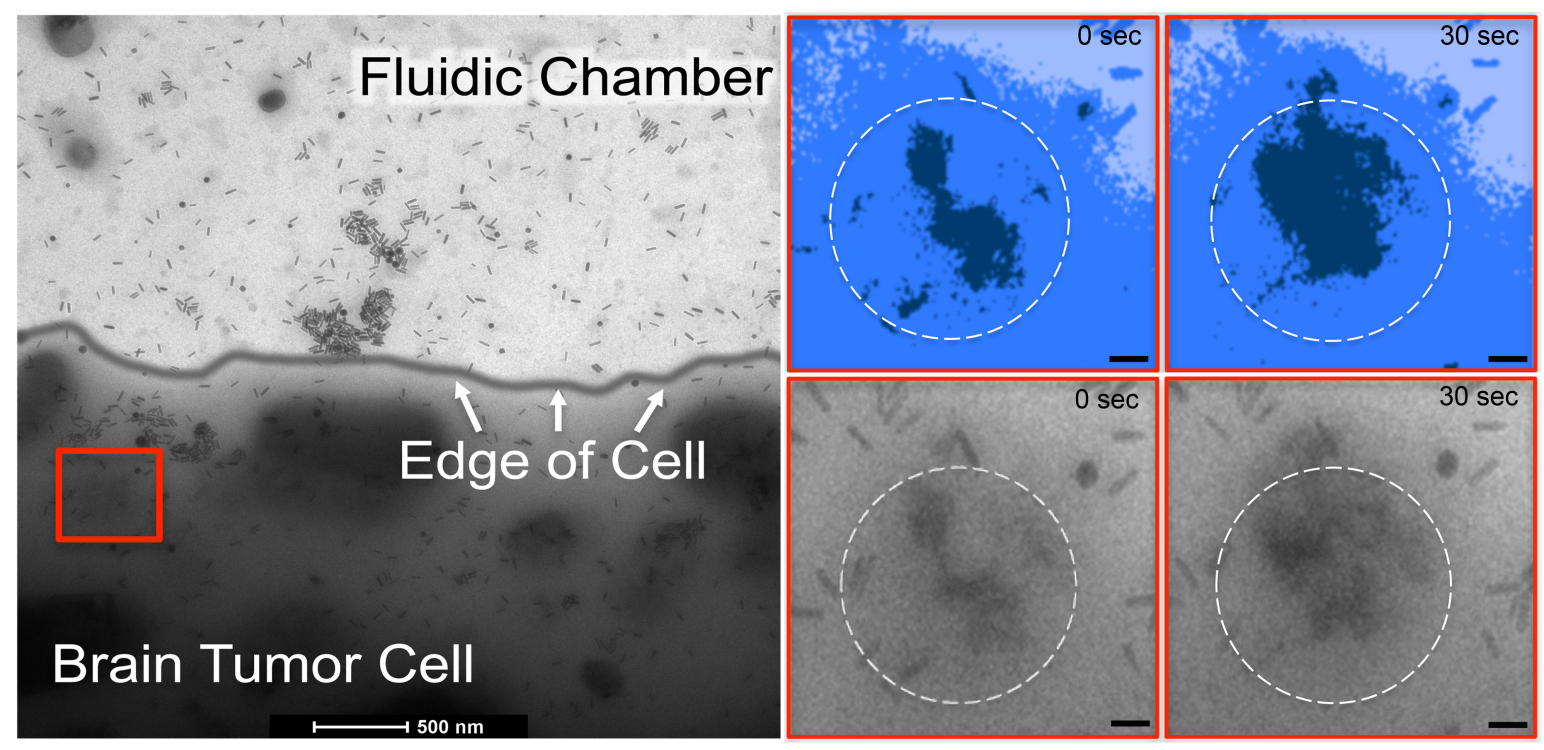

Figure 2. GS9-6/NOTCH1+ cells interacted with gold nanorods within 20-minutes of TEM imaging. Serial images were acquired for 30-second intervals within selected regions of interest (red square). Contour plots were generated for the initial and final time points, illustrating movements in the nanorods and solution (white dashed circles) within the tumor cells. Scale bars in side panels are $50 \mathrm{~nm}$. 